\title{
Hyperpathia and Aerobic Exercise
}

\section{J. C. Jiménez-Sáez ${ }^{1^{*}}$ (D) J. J. Jiménez-Rodríguez ${ }^{2}$ (D) and S. Muñoz ${ }^{2}$ (iD)}

${ }^{1}$ Department of Applied Physics in Aeronautical and Naval Engineering, ETSIAE, Polytechnic University of Madrid (UPM), Spain

${ }^{2}$ Department of Structure of Matter, Thermal Physics and Electronics, Faculty of Physical Sciences, Complutense University of Madrid (UCM)), Spain

*Corresponding author: J.C. Jiménez-Sáez, Department of Applied Physics in Aeronautical and Naval Engineering, ETSIAE, Polytechnic University of Madrid (UPM), 28040 Madrid, Spain, Tel: +34910675908

\begin{abstract}
The patient began to suffer from hyperpathia in his childhood. Then an electric shock-like pain indifferent support areas prevented the patient from putting pressure on these areas for a long period. The treatment with carbamazepine made the stabbing pain disappear. However, since then a pressure-induced soreness of varying intensity has developed in different body support areas if the stimulation on them exceeds a certain limit or if the dose of carbamazepine or the level of aerobic physical exercise decreases. This chronic pain in turn elevates the threshold pain so that a negative feedback can take place.
\end{abstract}

\section{Keywords}

Neuropathic Pain, Hyperpathia, Aerobic Exercise, Carbamazepine

\section{Introduction}

Hyperpathiais an abnormal painful sensation to a repetitive stimulus as well as an increased threshold pain. In general, hyperpathia is a clinical symptom associated with a certain neurological illness. In fact, there are several disorders such as central pain syndrome, complex regional pain syndrome or several peripheral neuropathies that present this symptomatology. However, unlike other studies [1], this article presents a case in which hyperpathia is really the only illness since no underlying physiopathology or psychopathology matches the symptomatology.

\section{Medical History}

The patient is a 51-year-old male of normal consti- tution with a pollen allergy and without significant surgery or disease. He has a case history of hypersensitivity to the pressure located on the main support areas of the body: Soles of the feet and lower part of the hipbone. This hypersensitivity gives rise to chronic pain if a long or brief but intense support occurs. Once a region is stimulated, the patient asserts that the painful sensation must be controlled and limited for quite some time since otherwise this could easily reach levels that would prevent support of the affected area for a long time. Besides, the patient also suffers from problems of hypersensitivity to many drugs associated with an abdominal pain at the height of the bladder.

The first symptom occurs in his childhood ( 13-years-old). Then, the patient experienced a severe neuropathic pain in the perineum area while seated on a bench. As a result, he had to sit on a rubber ring without supporting this region for several months. This disorder might have been perfectly diagnosed as a pudendal nerve entrapment, an illness reported for the first time several years afterwards [2]. The following set of symptoms appeared at the age of 17-years-old. After a long rest period without doing physical exercise, the patient developed pains in several areas: The back of both heels, both Achilles tendons and the bottom of both heel bones. In this last case, this disorder might be diagnosed as an entrapment of Baxter, an illness reported for the first time a few years later [3]. However, the piercing pain did not improve with non-steroidal anti-inflammatories or even worsened with the local injection of a steroid preparation.

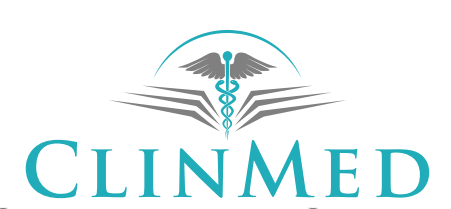

INTERNATIONAL LIBRARY

Citation: Jiménez-Sáez JC, Jiménez-Rodríguez JJ, Muñoz S (2020) Hyperpathia and Aerobic Exercise. Int J Sports Exerc Med 6:175. doi.org/10.23937/2469-5718/1510175 Accepted: September 11, 2020; Published: September 13, 2020

Copyright: (c) 2020 Jiménez-Sáez JC, et al. This is an open-access article distributed under the terms of the Creative Commons Attribution License, which permits unrestricted use, distribution, and reproduction in any medium, provided the original author and source are credited. 
At the age of 24 the patient was diagnosed with generalized hyperpathia and alteration of the pain threshold and he was prescribed with carbamazepine $(50 \mathrm{mg}$ daily). From his youth until now, this dose has increased and other areas have been sensitized, namely: both hips, both buttocks, both fore feet and both knees. In his daily life he should make use of a range of aids: Foams, spring folding chairs, or the car and its suspension to sit down; foams or spring folding chairs to kneel; viscoelastic urethane soles and polyamide socks to walk; and an innerspring mattress with a spring base to sleep.

\section{Medical Treatment and Tests}

Throughout his life a set of medical tests were performed on the patient without any significant pathological discovery: X-rays, computed tomography scanning, magnetic resonance imaging, bone scintigraphies, quantitative computed tomographies, biochemical tests and electromyography and nerve-conduction studies on sensory and motor nerves of both lower and upper limbs.

The tele thermology of buttocks and the soles of the feet offered findings that are more significant. Specifically, the thermograms of heels showed a noticeable pattern of hypoemission. This fact reflects the lack of functionality of the autonomic system against the thermicstimulation, which indicates that hyperpathia disables the local sympathetic function. In fact, the study concludes that both heels reflect an abnormal thermic pattern so that an intense autonomic hyperactivity is evidenced. This test will connect this disorder with the sympathetically maintained pains.

As for the medication, we highlight the effect of some drugs. Amitriptyline was initially prescribed together with carbamazepine; however, an in tolerance in the form of abdominal pain developed during its continual intake. Nevertheless, the patient does not remember any effect on his symptoms. The same happened with venlafaxine, duloxetine or fluoxetine. In his youth, the patient did not suffer from depression. However, he developed this illness due to the long disabling periods in bed. In this case, the patient takes paroxetine, which is well tolerated.

Different local anesthetics did not visibly improve the pressure-induced pain control either: The epidural administration of bupivacaine and the periodic intravenous infusions of lidocaine and ketorolac. Indeed, the patient mentions that he gave up this last treatment due to a strong and sustained pain in the lower part of the abdomen. Since then, he has suffered from intolerance to many medicines due to the recurrence of this pain. The medical diagnosis on this matter has been irritable bowel syndrome.

The last attempts were performed with antiepileptic drugs frequently prescribed for chronic pains. Lacosamide and lamotrigine cause him abdominalpain.
Oxcarbazepine causes him headache and although it derives from carbamazepine, it lacks its benefits. Finally, gabapentin and pregabalin were well tolerated at first, but affect his muscular system. In fact, use of the latter contributes to the pain control, but combined with physical exercise it caused him weakness and severe muscle contractures. The patient relates that his muscular strength was normal in his youth; however, in maturity he claims that he suffers from frequent muscular spasms and mild contractures when he increases physical activity.

\section{Discussion}

His family does not have a history of this disease; however, the father suffers from Parkinson's. This fact suggests the possible genetic origin of this disease. The treatment with carbamazepine has turned the paroxysmal pain into a persistent pain during support and he has almost completely got rid of allodynia and hyperalgesia. For now, no other medication has shown the effectiveness of this drug. In fact, carbamazepine, along with regular aerobic sport and a strict control of the duration and hardness of the different body supports allow the patient to live without any painful sensations. However, if the equilibrium among these factors is altered in an area, the chronic pain appears since the threshold decreases.

A clear origin would differentiate this illness from other similar pathologies. It begins with peripheral nerve compression, which makes it more similar to a compression neuropathy, and it produces pain in the compressed nerve during support just like radicular pain. In fact, in several areas the patient can point at the sore nerve. Besides, he is particularly sensitive to the support on edges or projections.

As mentioned above, the patient must exercise regularly, especially swimming. If he cannot move a leg for some time, the sensations of paresthesia begin first and after of pain appear in the support areas of this leg. Therefore, the exercise routine performs a peripheral pain modulation. It is known that the aerobic exercise stimulates peripheral nerve regeneration and accomplishes central pain modulation [4], in addition to promoting neurogenesis by increasing the production of neurotrophic factors [5]. In this case, the patient does not offer additional clues about the connection of sport with central mechanisms. He only mentions that the painful sensations are attenuated or temporarily inhibited during this activity. However, this might be due in part to those muscle nociceptors which are not excited under muscle contractions and stretching.

The patient also states that if a sheet simply rests on his buttocks or heels during sleep, the following day the pain sensations begin earlier in that area. Even, if this fact repeatedly happens, it can produce alterations in the local threshold. Therefore, a stimulus of very low in- 
tensity which a priori would not damage the peripheral nerves and that is inhibited during sleep by subcortical structures [6] can induce changes in the pain perception. This fact suggests that the central affectation of this disease is probably found at spinal level.

\section{Conclusions}

A novel medical case is reported in this article. The patient suffers from an abnormal and variable frontier between painful and non-painful pressure sensations. Thus, the patient can perceive pain for pressure stimuli whose duration or hardness exceed limits far below what a normal person experiences. Therefore, he must control these sensations in order to avoid their transformation into pain in all the body support areas. Besides, this threshold depends on the dose of carbamazepine and regular aerobic exercise. The patient also shows a great intolerance to all types of medication probably due to the same pathology since its intake causes him head or abdominal pain. The term that would best describe this illness would be a generalized hyperpathia.

\section{Acknowledgements}

This work was supported by the Complutense University of Madrid. (Bioelectromagnetism Group 910305).

\section{Conflicts of Interests/Financial Disclosures}

None.

\section{References}

1. Roberts WJ, Foglesong ME (1988) Spinal recordings suggest that wide-dynamic-range neurons mediate sympathetically maintained pain. Pain 34: 289-304.

2. Amarenco G, Lanoe $Y$, Perrigot M, Goudal H (1987) A new canal syndrome: compression of the pudendal nerve in Alcock's canal or perinal paralysis of cyclists. Presse Med 16: 399.

3. Baxter DE, Pfeffer GB (1992) Treatment of chronic heel pain by surgical release of the first branch of the lateral plantar nerve. Clin Orthop 279: 229-236.

4. Dobson JL, McMillanJ, Li L (2014) Benefits of exercise intervention in reducing neuropathic pain. Front Cell Neurosci 8: 102.

5. Szuhany KL, Bugatti M, Otto MW (2014) A Meta-Analytic Review of the Effects of Exercise on Brain-Derived Neurotrophic Factor. J Psychiatr Res 60: 56-64.

6. Foo H, Mason P (2003) Brainstem modulation of pain during sleep and waking. Sleep Med Rev 7: 145-154. 